\title{
Reform of EDA Course Design of Electronic and Information Using Model Based Designing Method
}

\author{
Wenmiao Song ${ }^{1, a}$, Hanbai Fan ${ }^{1}$ \\ ${ }^{1}$ School of Electronics and communication, North China Electric Power University, \\ Baoding; 071003, China; \\ awenmiaosong@tom.com
}

Keywords: MDL; FPGA; curriculum designing; matlab/DSPbuilder

\begin{abstract}
With the development of the modern electronics technology, the limitation of traditional method which only used VHDL language is more and more clear. To keep up with the newest development, we introduced method of model based design into the curriculum designing of the EDA course. In this paper we first introduced the idea of Model Based Design (MDL), the designing process, and an example based MDL using DSPbuilder modules in simulink software environment. At last, we design several projects according to the characteristics of major electronics and information .Compared with the previous schemes, the objective of this study is to design the experiment teaching system, with hardware and software function in system programmable, flexible design can be upgraded, cut, high reliability, low cost; experimental project of software has from simple to complex, from basic experiment gradually extended to the independent innovation of experimental features, accept the training of students can quickly master FPGA design methods, and fully mobilize students' innovative initiative, and maximally improve the learning interest of the students, cultivate students' capability of independent innovation.
\end{abstract}

\section{Introduction}

EDA course is professional undergraduate foundation courses of electronics, computer, automation and other information class professional in North China Electronics Power University. It is also some specialized courses necessary to placement program. Universities training must not only have a solid theoretical foundation, but also need solve practical problems and practice of practical ability using information technology tools. In past years, we have successfully integrated the traditional "digital electrical circuit and system design theory courses and EDA tools and hardware description language to complete the digital system design organically combined. With the development of electronic technology, a variety of large-scale programming integrated circuit s have been widely used, the limitations of traditional design method has become the obstacle to the programmable chip give full play to the performance, so method of hardware and software collaborative designing has become a new research direction . To make the students have the design concept of hardware and software cooperation, contact and understanding more advanced, more flexible system design method, the visual based model design concept [1] is introduced into the application of FPGA in future teaching and curriculum design.

\section{The Reform of Project Design by Introducing Idea of Model Based Designing}

\subsection{The idea of Model based designing}

The core of model based design is algorithm model, i.e., Users implement some of the ideas with Simulink or Builder DSP Blockset. Compared with the traditional software development process Model Based design is a process which enables developers more efficient and low costly. Model based designing covers a wide range of signal processing, control system, communication industry. Expression model of the system is the center of the whole development process, through demand, design, implementation and testing. Model itself is an executable specification, developers modify the optimization model is to design the repair, without the need to implement the test and verification 
system can through simulation in the early design period and to verify the correctness, and in the implementation phase, especially for embedded software, the code generated by the model. The detail designing flow of Model based designing is shown as Fig. 1.

MATLAB includes a powerful environment for dynamic systems simulation Simulink [2], which allows both digital and analog devices into simulation and has powerful tools to estimate simulation results. Simulink fully covers all developers' needs on this step of development and during the functional verification of FPGA algorithms. For automatic creation of HDL code MATLAB has a special add-on HDL Coder. It allows creating Verilog or VHDL code for FPGA designs based on a Simulink model without developer's participation. Using HDL Coder, the developer works only with the model while all stages of its transformation into an FPGA configuration file proceed automatically.

Model based designing allow rapid modeling and testing of algorithms, before committing to the expense of development for the final hardware platform, and the resulting cost benefits achieved by avoiding late changes to the design. Meanwhile, consistent behavior can be confirmed and demonstrated at all stages. The model design method is being widely concerned by the electronic design professionals. Nowadays, these advanced methods are used in Power Electronics [3], avionics, automotive, telecom and others.

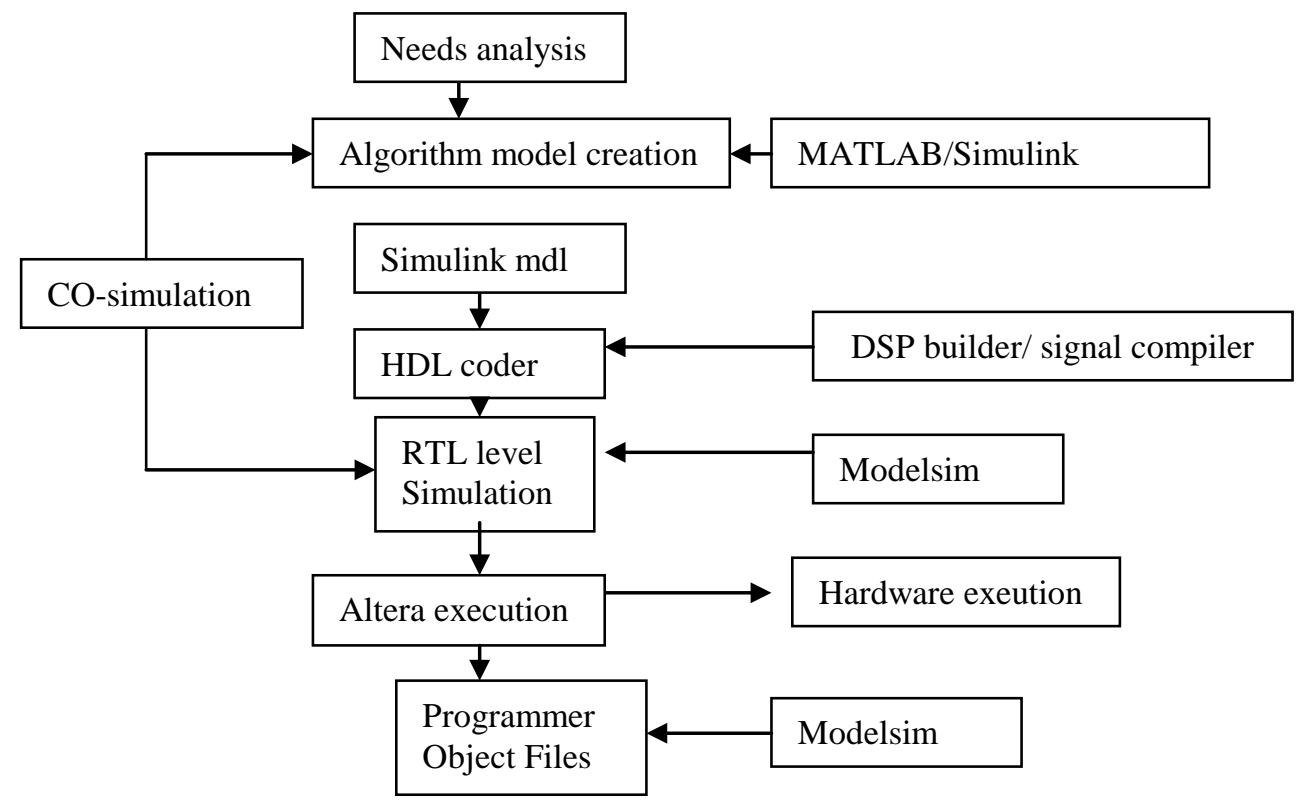

Fig. 1. The designing process of MDL

\subsection{The designing flow of the model based designing}

(1) Create a precise model of the algorithm

Simulink [4] is an oriented to multi-domain simulation and based on the model design of the frame diagram of the module environment. It supports the system level design, simulation, automatic code generation and embedded system of continuous testing and verification. MATLAB includes a powerful environment for dynamic systems simulation Simulink, which allows including both digital and analog devices into simulation and has powerful tools to estimate simulation results. Simulink fully covers all developers' needs on this step of development and during the functional verification of FPGA algorithms. Simulink provides a graphical editor, custom module library and solver, to carry out dynamic system modeling and simulation. Through integration with the MATLAB \& simulink[5], you can not only fusion the matlab algorithm to incorporated into the model, but also the simulation results of matlab to do further analysis. A digital down converter designed using DSP builder is shown as Fig. 2. 


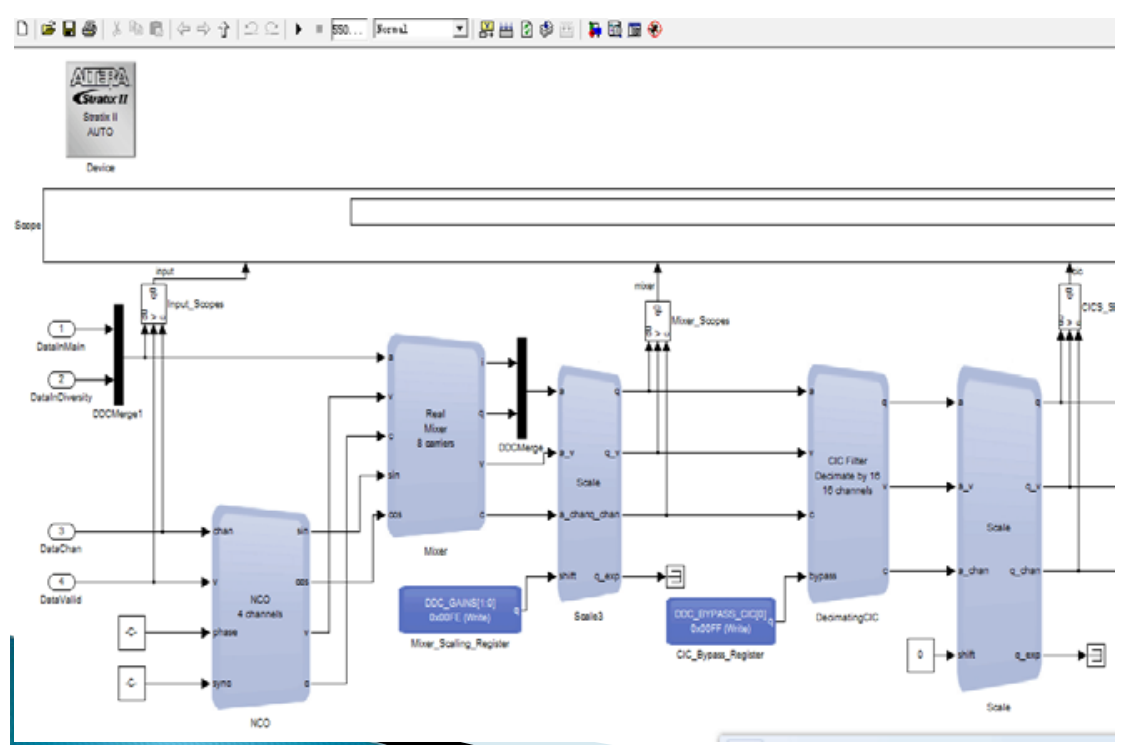

Fig. 2 DDC modular designed in Simulink

(2)Optimization designing with advanced modular library

DSP system developers will face a series of challenges and opportunities in the modern system design. Altera tools, IP core and reference design provide high efficiently developing process, thus reducing the risk of $\mathrm{R} \& \mathrm{D}$ of new products. DSPbuilder advanced module library is the tool to resolve the problems. Advanced DSP modular library is used easily as building blocks or simple pointing mouse. When we setup the QuartusII 11.0 and the DSPbuilder 11.0, we can open the matlab/simulink library and find the dspbuilder module library [3] which is shown in Fig. 3.

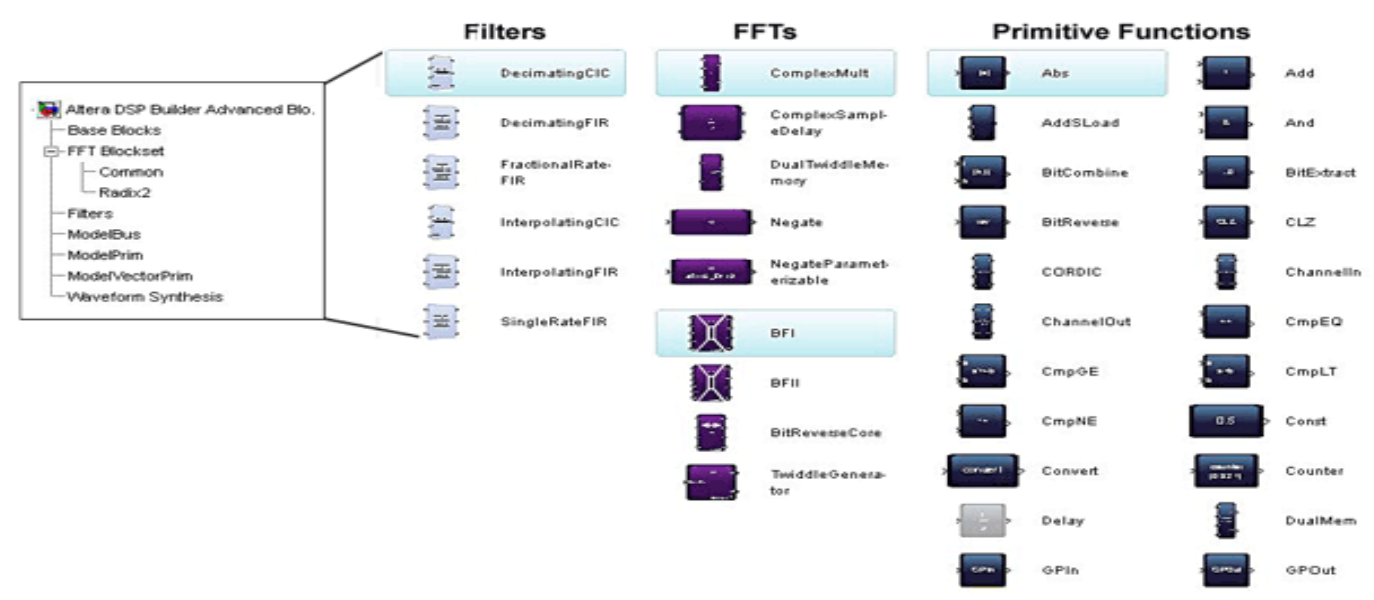

Fig. 3. Advanced DSPbuilder Blockset

Automatic code generation is an important part of the model design, which improves the quality of the algorithm code, and the advantage is that it can use the opening models and function of Matlab/simulink. Only a few simple modules or a few function statements can achieve complex functions, and through automatic code generation function will be simple model or function to generate complex HDL or C code algorithm statement .In Fig. 3, The modular” Run QuartusII” is a module which can convert the simulink model into QuartusII directly .

(3)Testify the designed project and programs

After simulating designed project in simulink, we can convert the user' designing in simulink into VHDL text or Verilog by 'Run Quartus II 'module .In Fig. 4 suitable parameter is setting according to the object board such as device type ,speed level, main clock etc .Then we can simulate in RTL level using testbench which be generated automatically. Finally we can program the designed module into the hardware board and running. We can justify the consistency result of hardware and the Simulink. And can use HIL to testify the real time and can accelerate simulation process. 

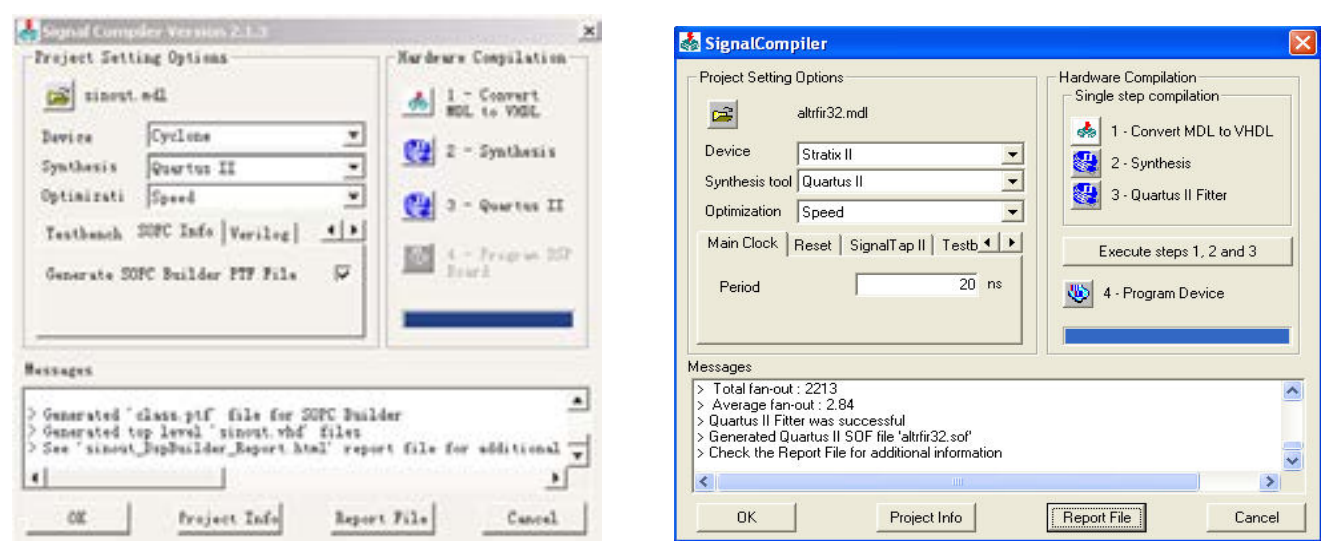

Fig. 4. Parameter setting of signal compiler

\section{The testbench: Altera DE2-115 Development and Education Board}

The DE2 series has consistently been at the forefront of educational development boards by distinguishing itself with an abundance of interfaces to accommodate various application needs. Extending its leadership and success, Terasic announces the latest DE2-115 that features the Cyclone IV E device. Responding to increased versatile low-cost spectrum needs driven by the demand for mobile video, voice, data access, and the hunger for high-quality images, the new DE2-115 offers an optimal balance of low cost, low power and a rich supply of logic, memory and DSP capabilities.

The DE2-115[4] adopts similar features from the earlier DE2 series primarily the DE2-70, as well as additional interfaces to support mainstream protocols including Gigabit Ethernet (GbE). A High-Speed Mezzanine Card (HSMC) connector is provided to support additional functionality and connectivity via HSMC daughter cards and cables. For large-scale ASIC prototype development, a connection can be made with two or more FPGA-based boards by means of a HSMC cable through the HSMC connector.

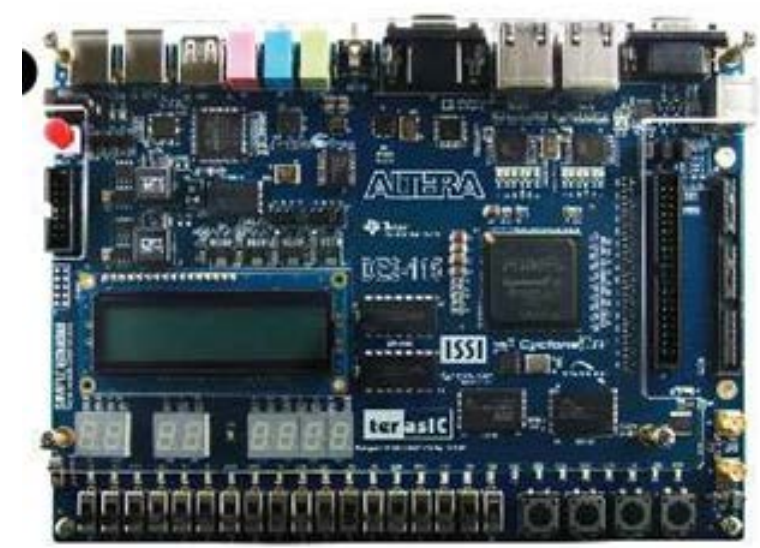

Fig. 5. DE-115 Hardware circuit board

The DE2-115 board has many features that allow users to implement a wide range of designed circuits, from simple circuits to various multimedia projects. To provide maximum flexibility for the user, all connections are made through the Cyclone IV E FPGA device. Thus, the user can configure the FPGA to implement any system design.

\section{Modified curriculum designing project based on MDL of electronics and information in NCEPU}

According to the characteristics of the major in electronics and information, we design six project designing based on MDL. All experimental project of software has from simple to complex, from basic experiment gradually extended to the independent innovation of experimental features. 
Project 1: the frequency divider designing which can divide the $50 \mathrm{Mz}$ main clock into $1 \mathrm{~Hz}$ output signal;

Project 2: IIR digital filter designing based on DSPbuilder;

Project 3: FIR digital filter designing based on DSPbuilder;

Project 4: Digital down converting module designing using MDL method;

Project 5: Design and implementation of SDR Based QPSK Modulator on FPGA;

Project 6: Model based design for power systems protection relays using Matlab/simulink

Project 1 and project 2 are basic tests whose purpose is to grasp the software environments and the whole designing flow including software and hardware designing and simulation. Project3 and Project 4 are mid-level item digital signal process by designing FIR and IIR filter. According to our major of electronics and information, we design project 5 correspond with wireless communication. Through the design of a SDR platform using Model based designing, we can enhance perceptual knowledge of wireless communication principles for. In order to expand the employment chance for graduates of electronic specialty, our final design question is about power electronics. Project6 will

Design Power Systems Protection Relays based on simulink. By implementing this project, undergraduate can combine the electronics designing with electric power communication [6].

\section{Conclusion}

Advanced concepts based on model design are deeply rooted in the hearts of the people, and it has been widely used. We design six projects using model based designing in our EDA course design according to the characteristics of electronics and information major. The designed six experimental projects have the features gradually from simple to complex, from basic experiment gradually extended to the independent innovation of experimental. Students can understand the idea of Model based designing and learn the designing flow from software simulation to hardware simulation. Finally they can get result by hardware programs. They can understand practically the professional course such as EDA, digital signal process, communication theory. Undergraduate accepting the training can quickly master FPGA design methods, and fully mobilize students' innovative initiative, and maximally improve the learning interest of the undergraduate, cultivate undergraduate' capability of independent innovation.

\section{References}

[1] Liu jie, Model_based designing for Qsys[M] (In Chinese),China machine Press, Beijing,2012.

[2] Venkataramani, Girish, et al. "Model-Based Hardware Design." Proceedings of the International Conference on Computer-Aided Design. IEEE Press, 2013.

[3] U. Meyer-Baese, A. Vera, A. Meyer-Baese, M. Pattichis, and R.Perry, "DSP with FPGAs: A XILINX/Simulink-based course and laboratory,” in Proc. SPIE Int. Soc. Opt. Eng., Mar. 2008, pp. 907-1-697 907-12.

[4] Kierby, B. Model Based Design for Power Systems Protection Relays, Using Matlab \& Simulink [C]. Developments in Power System Protection, 2008. IET 9TH International Conference on. 2008: $654-657$

[5] LIU Wei-lian,JIANG Xiu. A Design of Harmonic Detection in Power System Based on Xilinx FPGA [J]. Electrical Measurement \& Instrumentation, 2014, 51(10):2-6

[6]Juergen Wassner, Stephen Dominiak, Javier Moya Paya, Model based design of an avionics power line communications physical layer[C]. 2015 IEEE/AIAA 34th Digital Avionics Systems Conference (DASC), 2015, 9:1-11 